\title{
Visual impairment in very low birthweight children
}

\author{
Andrew Powls, Nicola Botting, Richard W I Cooke, Gail Stephenson, Neil Marlow
}

\begin{abstract}
Aims-To compare the visual function of a cohort of very low birthweight (VLBW) children in early adolescence with that of their normal birthweight peers; to correlate visual impairment in this group with available perinatal data; and to examine the relation between the visual ability of VLBW children and their cognitive and motor skills.

Methods-As part of a long term neurodevelopmental study, 137 VLBW children and 163 normal birthweight controls were visually assessed between the ages of 11 and 13 years. Their eyes were examined for strabismus and movement disorders, and the use of visual correction for refractive errors was noted. Measures were made of visual acuity, stereopsis, and contrast sensitivity. All children had standardised tests of motor ability and cognitive skills. Perinatal data, including cranial ultrasonography results, had been obtained from the children's notes. No data were available however, regarding retinopathy of prematurity as screening was not established when these infants were born.
\end{abstract}

Results-On all measures, the visual function of the VLBW children was poorer than that of the controls. Reduced visual function was present in $63.5 \%$ of VLBW children compared with $36 \%$ of controls. Poor contrast sensitivity and strabismus were predictive of poor motor skills in the VLBW children. Poor contrast sensitivity and poor visual acuity (at 0.3 metres) were predictive of lower IQ. Low birthweight, intraventricular haemorrhage, intrauterine growth retardation and low 1 minute Apgar scores predicted reduced visual function.

Conclusions-VLBW children have a high incidence of impaired vision. Stereopsis and contrast sensitivity are useful additions to the screening of this high risk group. They identified impaired vision that was not detected by normal screening and were related to impaired neurodevelopmental outcome.

(Arch Dis Child 1997;76:F82-F87)

Keywords: visual function; very low birthweight; cognitive impairment; motor skills; stereopsis; contrast sensitivity.

Several studies have shown that infants of very low birthweight (VLBW) are at increased risk of long term visual impairment. ${ }^{1-11}$ This risk is even greater among those infants who developed retinopathy of prematurity (ROP) during the neonatal period. ${ }^{12-15}$ ROP, however, is not the only risk factor for poor visual outcome; intraventricular haemorrhage (IVH) and periventricular leucomalacia (PVL) have also been associated with ocular morbidity. ${ }^{2}{ }^{316}$ Increased ambient light within the neonatal unit and phototherapy have also been proposed as possible adverse factors in retinal development. ${ }^{18} 19$

In the worst instances blindness may occur secondary to cicatricial $\mathrm{ROP}^{15}$ or intracerebral haemorrhage affecting the visual centres. ${ }^{16}$ Less severe visual impairments, including reduced visual acuity, refractive errors, strabismus, and nystagmus have been found in larger numbers of VLBW children ${ }^{1-15}$ than in normal birthweight children. The reported incidence of these visual impairments varies from $15-33 \%,{ }^{2}$ depending on the visual measures used, the age at testing, and the entry criteria.

More recently, a few reports have noted the presence of more subtle impairments of visual function among VLBW survivors of neonatal intensive care. These have included abnormalities of colour vision, ${ }^{1}$ stereo-acuity, ${ }^{15}$ and contrast sensitivity. ${ }^{18}$ These abnormalities may be present even in those children with otherwise apparently normal visual function. These studies have therefore found a higher incidence of overall visual impairment of $45-59 \% .{ }^{15}$ It is not clear from these studies whether these subtle impairments are related to adverse perinatal events, although one study of more mature infants implicated high levels of illumination in the nursery to impaired contrast sensitivity. ${ }^{18}$ Nor is it clear whether such subtle abnormalities are relevant to neurodevelopmental outcome.

This study examines the ocular morbidity and visual function, at 11-13 years, of a large cohort of VLBW children, for whom detailed perinatal data including cranial ultrasonographic studies were available.

\section{Methods}

The population studied was derived from two hospital based cohorts of VLBW children treated at the Mersey regional neonatal unit. Both groups have already been part of separate neurodevelopmental follow up studies, and have been combined in the present study to increase the numbers for analysis. The first group were children with a birthweight of $<1251$ g were born between January 1980 and June 1981 inclusive. These children had been 
seen at 6 and 8 years by Marlow and colleagues. ${ }^{20}$ The second group were children with a birthweight of $<1501 \mathrm{~g},<31$ weeks gestation, and birth rates between January 1982 and November 1983 inclusive. These children had been seen at 6 years of age. ${ }^{21}$ None of the children had a major neurodevelopmental handicap, and at the time of the original study were in mainstream schools. Altogether at the time of the six year studies, 146 VLBW children were recruited. For the present study, all the VLBW children were traced, but three children have since emigrated and five families were not willing to participate in a further study.

A normal birthweight control population was recruited, at the time of the initial studies, from classmates of the same sex and similar age to the VLBW children. This was found at the time to provide close matches for socioeconomic as well as educational variables. These original controls were also traced and, where possible, used for the present study. As the overall study also looked at educational and cognitive outcome, VLBW children who were now at different schools from their controls, had a new control selected from their present school to match for educational experience. Overall, 160 control children were assessed, of which 100 were original controls.

Informed written consent was obtained from the parents of all the children involved in the study. The children were seen and assessed in their schools. Further information was also sought from the parents in the form of a health questionnaire.

All assessments were made with the children wearing their usual visual correction, where appropriate. It was not possible within the scope of this study to refract the children to assess the appropriateness of their prescriptions.

Monocular visual acuity was assessed in each eye using Snellen charts at 6 metres and 0.3 metres. Also noted was whether the glasses worn were for correction of myopia or hypermetropia. Acuity was considered abnormal if either eye had an acuity of $6 / 12$ or worse.

Cover testing for was performed for the detection of strabismus. When present, the side and nature of the strabismus were noted. Examination for ocular movements was also performed.

Stereopsis was assessed using the TNO random dot test. ${ }^{22}$ The test pictures are viewed through red/green lenses worn over the children's glasses where appropriate. The test identifies the level of stereo-acuity between 30 and 480 seconds of arc. Normal stereo acuity on this test is defined as a resolution of $60 \mathrm{sec}-$ onds of arc or better, and reduced stereo acuity as a resolution of 90 to 480 seconds of arc.

Contrast sensitivity was tested at 18 inches using the Functional Acuity Contrast Test (FACT).$^{23}$ The contrast threshold for each eye is tested at five spatial frequencies: $1.5,3,6,12$ and 18 cycles per degree (CPD). The contrast threshold is considered abnormal if it is higher than the 95th centile of the population on which the test was standardised.
Gross and fine motor skills were assessed using age band 4 of the Movement Assessment Battery for Children (Movement ABC). ${ }^{24}$ As this test is used to identify motor impairment, higher scores represent poorer performance. A score of 13.5 or greater out of a possible total of 40 corresponds to the performance of the least able $5 \%$ of the population, thus it is considered to represent clinically significant motor impairment.

The children's IQ was measured using a short form of the Wechsler Intelligence Scale for Children (WISC III). ${ }^{25}$ This provides subscale measures of the verbal and performance elements of IQ as well as an overall, or full scale IQ.

The following perinatal variables had been recorded for the VLBW children and were used to examine the relation between adverse perinatal factors and visual impairment: Birthweight $(\mathrm{kg})$; gestational age at birth (weeks); small for gestational age $(>2$ SD below mean for gestation $)^{26}$; extremely low birthweight (ELBW <1000 g); fetal distress before or during labour; low Apgar scores at 1 and 5 minutes ( $<=2$ and $<=5$, respectively); fits; intraventricular haemorrhage (excluding subependymal haemorrhage); cystic periventricular leucomalacia; confirmed episodes of sepsis; respiratory distress syndrome (defined using clinical and radiological criteria).

The results were analysed using SPSS for Windows. The numbers of children were analysed using $\chi^{2}$ tests, and to predict visual impairment from perinatal data multiple logistic regression models were used.

\section{Results}

A total of 137 VLBW children were visually assessed. They had a median birthweight of $1100 \mathrm{~g}$ (range 620-1500 g) and a mean gestation of 28 weeks (range 24-35weeks). All children had been admitted to the Mersey Regional Neonatal Unit and 84 children had required mechanical ventilation. One hundred and sixty three control children were also visually assessed, although three did not have contrast sensitivity measured due to unavailability of the chart on the days of their assessments. Health questionnaires were received from 130 (95\%) of the VLBW children's parents and from $150(94 \%)$ of the control children's parents. Median age (range) at assessment was 142 months (132-163) for the VLBW children and 143 months (130-164) for the controls.

The VLBW children had poorer vision than their normal birthweight controls. The questionnaire revealed that 49 of $130(38 \%)$ VLBW children had failed an eye test at some point in their lives in contrast with 35 of $150(23 \%)$ of the controls $\left(\chi^{2}=6.84, P=0.009\right)$. More of the VLBW children, 41 of 130 (31\%), had worn glasses at some point compared with 29 of 150 (19\%) normal birthweight children $\left(\chi^{2}=5.53\right.$, $\mathrm{P}=0.02)$. Of the children still wearing glasses for refractive errors at the time of examination, there were more VLBW children than controls wearing correction for myopia. There were no differences in the number of children in each 
Table 1 Prevalence in numbers (\%) of visual impairments in VLBW children and normal birthweight controls

\begin{tabular}{lllll}
\hline & $V L B W(n=137)$ & Controls $(n=163)$ & $\chi^{2}$ & Pvalue \\
\hline Abnormal visual acuity: & $23(17)$ & $13(8)$ & & \\
$\quad 6 \mathrm{~m}$ & $19(14)$ & $10(6)$ & 5.47 & 0.02 \\
$0.3 \mathrm{~m}$ & $13(9.5)$ & $4(2.5)$ & 6.0 & 0.025 \\
Strabismus & $31(23)$ & $21(13)$ & 4.79 & 0.009 \\
Spectacle wear & $20(15)$ & $13(8)$ & 3.25 & 0.07 \\
$\quad$ Myopia & $11(8)$ & $8(5)$ & 1.18 & 0.27 \\
$\quad$ Hypermetropia & $50(36)$ & $24(15)$ & & \\
Stereopsis: & $27(20)$ & $9(5.5)$ & 18.9 & 0.00001 \\
Abnormal & $23(17)$ & $8(5)$ & 14.1 & 0.0002 \\
Absent & $25(18)$ & $6(4)$ & & \\
Poor contrast sensitivity & at spatial frequency: & $19(12)$ & 10.9 & 0.0009 \\
1.5 CPD & $44(32)$ & $20(12.5)$ & 18.5 & 0.00005 \\
3 CPD & $51(37)$ & $25(16)$ & 24.8 & $<0.00002$ \\
6 CPD & $62(45)$ & $31(19)$ & 31.3 & $<0.00000$ \\
12 CPD & $66(48)$ & 27.8 & $<0.00000$ \\
18 CPD & & &
\end{tabular}

Table 2 Prevalence of impaired motor performance, as assessed by the Movement ABC, in $V L B W$ children with impaired visual function

\begin{tabular}{|c|c|c|c|c|}
\hline & \multicolumn{2}{|c|}{$\begin{array}{l}\text { Prevalence of motor impairment ( } A B C \text { score } \\
>13 \text { ) by group }\end{array}$} & \multirow[b]{2}{*}{$\chi^{2}$} & \multirow[b]{2}{*}{ P value } \\
\hline & Impaired & Normal & & \\
\hline \multicolumn{5}{|l|}{ Acuity: } \\
\hline at $6 \mathrm{~m}$ & $12 / 23(56 \%)$ & $23 / 112(20 \%)$ & 9.9 & 0.002 \\
\hline at $0.3 \mathrm{~m}$ & $10 / 19(53 \%)$ & $26 / 117(22 \%)$ & 7.8 & 0.005 \\
\hline Squint & $8 / 13(61 \%)$ & $27 / 122(22 \%)$ & 9.4 & 0.002 \\
\hline Refractive error & $14 / 31(45 \%)$ & $22 / 105(21 \%)$ & 7.2 & 0.007 \\
\hline Stereopsis & $20 / 50(40 \%)$ & $15 / 85(18 \%)$ & 8.2 & 0.004 \\
\hline \multicolumn{5}{|c|}{ Contrast sensitivity ( $C P D=$ cycles per degree): } \\
\hline at $1.5 \mathrm{CPD}$ & $9 / 21(43 \%)$ & $27 / 114(24 \%)$ & 3.3 & 0.07 \\
\hline at $3 \mathrm{CPD}$ & $10 / 23(43 \%)$ & 26/112 (23\%) & 4.0 & 0.04 \\
\hline at $6 \mathrm{CPD}$ & $21 / 40(52 \%)$ & $15 / 95(16 \%)$ & 19.4 & $<0.0000$ \\
\hline at $12 \mathrm{CPD}$ & $21 / 44(48 \%)$ & $15 / 91(16 \%)$ & 14.8 & 0.0001 \\
\hline at $18 \mathrm{CPD}$ & $24 / 50(48 \%)$ & $12 / 85(14 \%)$ & 18.4 & $<0.0000$ \\
\hline
\end{tabular}

group wearing correction for hypermetropia (table 1) .

Examination showed that there was a higher incidence of strabismus among the VLBW children (table 1). Thirteen VLBW children had strabismus, of these most were esotropic, three right-sided and eight left-sided. Two children had alternating strabismus. Among the controls only four children had strabismus. Three children were esotropic, two left-sided and one right The remaining child was exotropic. Only one VLBW child and no controls were found to exhibit nystagmus.

Visual acuity, both distant (6 metres) and near ( 0.3 metres), was poorer in the VLBW children than in their controls, despite the children wearing their usual corrective lenses. One VLBW child was blind in one eye (visual acuity worse than $6 / 60$ ). Overall, there were more VLBW children than controls with impaired visual acuity (6/12 or worse) for both measures.

Table 3 Prediction of visual morbidity from perinatal data

\begin{tabular}{|c|c|c|c|}
\hline Visual impairment & Perinatal factor & Odds ratio $(95 \% \mathrm{CI})$ & $P$ value \\
\hline $\begin{array}{l}\text { Visual acuity }<6 / 9 \\
\text { at } 6 \mathrm{~m} \\
\text { at } 0.3 \mathrm{~m}\end{array}$ & $\begin{array}{l}\text { Birthweight }(\mathrm{kg}) \\
\text { No predictive variables }\end{array}$ & $-20.1(-1.8$ to -221.4$)$ & 0.014 \\
\hline $\begin{array}{l}\text { Poor contrast sensitivity (at } \\
\text { any spatial frequency) }\end{array}$ & Birthweight $(\mathrm{kg})$ & $-6.7(-1.1$ to -40.4$)$ & 0.035 \\
\hline Myopia & $\begin{array}{l}\text { Birthweight (kg) } \\
\text { SGA } \\
\text { IVH }\end{array}$ & $\begin{array}{l}-99(-3.3 \text { to }-2980) \\
5.4(1.3 \text { to } 22.8) \\
4.6(1.1 \text { to } 19.3)\end{array}$ & $\begin{array}{l}0.007 \\
0.02 \\
0.03\end{array}$ \\
\hline Hypermetropia & No predictive variables & & \\
\hline Squint & IVH & $5.1(1.2$ to 21.5$)$ & 0.02 \\
\hline Absent stereopsis & Apgar score (1 minute) & $-1.3(-1.1$ to -1.16$)$ & 0.003 \\
\hline
\end{tabular}

SGA: Small for gestational age; IVH: intraventricular haemorrhage. (table 1). Even among those children with visual acuity of $6 / 9$ or better we found that the VLBW children had significantly lower acuity than their controls ( $\mathrm{P}=0.004$, Mann-Whitney $\mathrm{U}$ test). Impaired distant visual acuity was present more frequently in the left eye of the VLBW children than in the right. The reason for this finding was unclear.

Stereopsis was reduced or absent in significantly greater numbers of the VLBW children than in those of normal birthweight (table1).

The VLBW children had significantly poorer contrast sensitivity than the controls; this was true for both eyes and for all grating widths (table 1). However, the differences were more obvious for the left eye and for the smaller grating widths (higher spatial frequencies). No child had abnormalities of contrast sensitivity exclusively at the lower spatial frequencies. Two index children were unable to complete the test. One of these children was blind in one eye with substantially impaired vision in the other eye. The other child failed to complete the test for cognitive reasons.

VLBW children with reduced visual function on any measure, were more likely to have significantly impaired motor skills, as assessed by the Movement ABC (table 2). A multiple logistic regression model was used to see which visual outcome measures were most predictive of significantly impaired motor skills. Reduced contrast sensitivity, at any spatial frequency, proved the best predictor, the model fitting best for reduced contrast sensitivity at 12 cycles per degree. The odds ratio for significantly impaired motor performance among those failing at this spatial frequency was $8.4 \quad(95 \%$ confidence interval 2.85 to 24.7$) ; \mathrm{P}=0.0001$. The presence of a squint was also predictive of poor motor skills with an odds ratio of 5.1 (1.36 to 19.1 ); $\mathrm{P}=0.013$. Poor visual function was also predictive of lower IQ. A similar multiple logistic regression model was used to predict a full scale IQ more than one standard deviation below the mean $(<85)$. Abnormal contrast sensitivity was the only significant predictor of low IQ, the best fit being for reduced contrast sensitivity at a spatial frequency of 18 cycles per degree. The odds ratio for a full scale IQ of $<85$ among those failing at this spatial frequency was 2.6 (1.2 to 5.9); $\mathrm{P}=0.014$. This prediction was more significant if the performance IQ subscale was used as the dependent variable in the model: odds ratio 4.2 (1.4 to 12.8$) ; P=0.009$. Abnormal contrast sensitivity was not predictive of the verbal IQ subscale score, but this score was significantly predicted by poor visual acuity (at 0.3 metres) with an odds ratio of 2.1 (1.3 to 3.4); $\mathrm{P}=0.002$.

Multiple logistic regression models were used to determine whether these measures of reduced visual function could be predicted from the available perinatal data. The perinatal variables used in the model were as follows: Variables relating to growth and maturity: birthweight (kg); gestation (weeks); small for gestational age; extremely low birthweight; Variables relating to perinatal asphyxia or other risk factors for neurological damage: fetal distress; low Apgar scores at 1 and 5 minutes $(=<2$ and 
$=<5$, respectively); fits in the neonatal period; intraventricular haemorrhage; cystic periventricular leucomalacia;

Variables relating to other neonatal diseases: sepsis; respiratory distress syndrome (RDS). It should be noted that screening for ROP had not been established in this unit during this period.

Poor visual acuity and abnormal contrast sensitivity were both significantly predicted by low birthweight alone. The presence of myopia was predicted by low birthweight, low weight for gestation (SGA), and by intraventricular haemorrhages. Strabismus was predicted by intraventricular haemorrhage alone. Absent stereopsis was predicted by low 1 minute Apgar score. The odds ratios and significance values are shown in table 3 . No predictive variables were found for hypermetropia or for visual acuity at 0.3 metres, but the numbers of children with these problems were small.

\section{Discussion}

Abnormalities of visual function were detected more frequently among the VLBW children than their controls for all measures used. In the VLBW group 33\% had abnormalities that could be detected by standard visual screening measures-acuity testing, cover testing, and refraction. This incidence is similar to that reported by Keith and Kitchen. ${ }^{7}$ Comparison of the overall incidence of visual impairment with the findings of other studies is, however, problematic. There are major differences between the various studies of visual morbidity in VLBW children, both in the range of measures used and in the entry criteria, with some studies including children with major neurological handicap. Gibson et at found a much lower incidence $(15 \%)$, but their study was conducted on infants under 12 months old, and this may be too early to detect some abnormalities of visual function which may develop subsequently, including myopia ${ }^{4}$ and strabismus. ${ }^{5}$

Individual tests are easier to compare. We found reduced monocular visual acuity (6/12 or worse) in $17 \%$ of the VLBW group. This is higher than in the studies of McGinnity et $a l^{4}$ and Gibson et al ${ }^{2}(10.5 \%, 9.5 \%)$, however both these studies reported binocular visual acuity which may account for the lower incidence. In keeping with the study by Sebris et $a l,{ }^{10}$ we found that even in the children with acuities in the normal range, the VLBW children had significantly lower acuities.

Strabismus was detected in $9.5 \%$ of our VLBW group. Previous studies have reported an incidence of 9.9-25\%, ${ }^{2-7}{ }^{15}$ with a higher incidence in children who had ROP. ${ }^{13}$ Our figures are at the lower end of this range and there may be a number of reasons for this. Firstly, our study excluded children with cerebral palsy, a group with a high incidence of strabismus. ${ }^{127}$ Secondly, the older age at examination may have missed some squints treated at an earlier age, and this may be reflected in the higher incidence of reduced or absent stereopsis.

Visual correction for myopia was worn by $15 \%$ of our VLBW group, which is comparable with that found in previous studies that have reported an incidence of myopia of $10-27 \%,{ }^{5-8} 121415$ with a higher incidence in the subgroup who had ROP. ${ }^{1214}$ As our study relied on the numbers of children wearing visual correction, there may have been a number of children in our study with undetected refractive errors.

Among the children who would have passed these standard screening tests, we found a further $30 \%$ of the group to have reduced visual function on the more subtle tests, stereopsis, and contrast sensitivity. This finding is in keeping with that of Dowdeswell et al, ${ }^{1}$ who found a significant reduction in contrast sensitivity and stereopsis along with abnormalities of colour vision among premature children of less than 32 weeks gestation. When we include the outcome of these tests, we found $63.5 \%$ of the VLBW group had measurable abnormalities of visual function compared with $36 \%$ of controls.

The VLBW children in our cohort had a higher incidence of neurodevelopmental impairments, both in motor ${ }^{28}$ and cognitive areas (unpublished data). In this study we have demonstrated strong links between these abnormal outcomes and reduced visual function, particularly in relation to motor skills, IQ, maths and reading ability. These associations were strongest in children with strabismus and those with reduced contrast sensitivity. This is in contrast to Dowdeswell et al, ${ }^{1}$ who found impaired contrast sensitivity in children without neurological impairment (or ROP), but this may reflect the more sensitive measures of neurodevelopment used in our study. There are two possible explanations for associations between visual and neurodevelopmental impairments. Firstly, both areas of disability may have a common aetiology, a consequence of neurological damage. Alternatively, poor visual function may directly affect the development of motor and cognitive skills.

Several studies have reported an association between visual impairment and major neurodevelopmental handicap in VLBW children with cerebral lesions detected by ultrasonography and magnetic resonance imagingspecifically intraventricular haemorrhage and cystic periventricular leucomalacia involving the parietal and occipital areas. ${ }^{16}{ }^{17}$ Our study found similar links between intraventricular haemorrhage and the presence of strabismus, which in turn was associated with poor motor skills. We also found that low 1 minute Apgar scores and growth retardation were associated with absent stereopsis and myopia, respectively. Both these variables are related to fetal compromise in utero and are risk factors for ischaemic brain damage. These findings lend support to a common aetiology with motor impairment. We found no association, however, between intraventricular haemorrhage and periventricular leucomalacia and reduced contrast sensitivity, which was the most sensitive predictor of impaired motor and cognitive function in our cohort. Although this latter finding does not seem to support a common aetiology, the neurodevelopmental impairments studied in our cohort are of a more sub- 
tle nature than the handicaps reported in the previous studies. They may, therefore, still be associated with more subtle lesions in the periventricular white matter not detected by the low resolution ultrasonography available in 1980-3. Magnetic resonance imaging scans have not yet been performed on our cohort.

The relatively weak association between visual impairments and neurological lesions in our group may, however, support the alternative hypothesis, that there is a direct, causative link between poor visual function and abnormalities of neurodevelopment. The age of our cohort means that at the time of their treatment as neonates there was no regular ophthalmological screening. Without data on ROP in this group, our ability to examine this hypothesis is limited. Our study does provide, however, some evidence in support of this hypothesis. Poor motor skills in our VLBW children were best predicted by reduced contrast sensitivity at the higher spatial frequencies. Abnormalities at these spatial frequencies are associated more with retinal pathologies rather than cerebral lesions, which preferentially affect contrast sensitivity at the lower spatial frequencies. ${ }^{18}{ }^{23}$ Additionally, the areas of neurodevelopment most significantly predicted by lower contrast sensitivity in our study-motor skills and performance IQ - are those where visual input or visuo-spatial ability are important.

Abnormalities of visual attentiveness and visually guided behaviour have been described in children with poor contrast sensitivity. ${ }^{29}$ In addition, improvement of contrast sensitivity by refractive correction improved these behavioural problems, even where there was no measurable improvement in acuity. Such behavioural abnormalities may be a mechanism whereby subtle visual impairment may adversely affect the development of motor and cognitive skills.

As the VLBW children were not screened ophthalmolgically while infants, we cannot determine how much of the long term abnormalities of visual function are the result of retinopathy or other ocular insults in the neonatal period. Several studies have shown a higher incidence of visual impairments in children with ROP as infants. ${ }^{12-15}$ These impairments described are similar to the types of problems seen in our cohort: reduced acuity; strabismus; and myopia. Our finding of an association between an increasing incidence of visual impairment with decreasing birthweight may relate, in part, to the inverse relation between birthweight and the incidence of ROP.

Retinopathy, however, is not solely responsible for ocular damage in the neonatal period. Very premature infants have a greater exposure to other adverse factors, some of which have been implicated in the aetiology of impaired vision. These factors include altered environmental temperature, ${ }^{19}$ high ambient lighting and phototherapy, ${ }^{18} 19{ }^{30}$ impaired nutrition and prolonged oxygen dependency. Premature birth itself may adversely affect visual maturation. ${ }^{19}{ }^{29}$

The exposure of our cohort to these various adverse factors cannot be accurately assessed retrospectively. No measures of lighting levels were made at the time of our cohort's treatment, but they will however, have been exposed to high ambient lighting day and night while in the intensive nursery and many will have received phototherapy, although standard eye patching was used. Very premature infants are inevitably exposed to impaired nutrition; total parenteral nutrition was used frequently among the very immature in our cohort, although lipid was used less frequently. For those able to take enteral nutrition, breast milk will have been used in most cases as there was a breast milk bank operating at the time. No data on the presence of bronchopulmonary dysfunction were available, and we failed to find any association between impaired vision and the length of artificial ventilation or the presence of surfactant deficient lung disease in our cohort.

We feel that the tests for stereopsis and contrast sensitivity are useful additions to the standard battery of visual screening tests for VLBW children, who are at high risk of visual impairment. The use of these tests in high risk groups, such as children of very low birthweight, has been recommended elsewhere. ${ }^{29}$ They are easy to perform and identify a group of children with reduced visual function that is not identified using the standard tests. They may help identify a group at risk of poor neurodevelopmental outcome and may be more useful in predicting such problems than the standard visual tests. Further work needs to be done to determine whether this association is causal and if so, to see if early intervention with refractive correction helps to improve neurodevelopmental outcome.

We acknowledge the secretarial help of Mrs S Longworth and Mrs D Bolger and the schools who gave their time and facilities for our assessments.

This project was funded by the Medical Research Council.

1 Dowdeswell HJ, Slater AM, Broomhall J, Tripp J. Visual deficits in children born at less than 32 weeks gestation with and without major ocular pathology and cerebral damage. Br f Ophthalmol 1995;79:1-6.

2 Gibson NA, Fielder AR, Trounce JQ, Levene MI. Opthalmic findings in infants of very low birthweight. Dev Med Child Neurol 1990;32;7-13.

3 McGinnity FG, Halliday HL. Perinatal predictors of ocular morbidity in school children who were low birthweight. Paediatr Perinatal Epidemiol 1993;7:417-25.

4 McGinnity FG, Bryars JH. Controlled study of ocular morbidity in school children born preterm. $\mathrm{Br} \mathcal{F}$ Ophthalmol 1992;76:520-4.

5 Burgess P, Johnson A. Ocular defects in infants of extremely low birthweight and low gestational age. $\mathrm{Br} \mathcal{F}$ Ophthalmol 1991;75:84-7.

6 Gallo JE, Lennerstrand G. A population based study of ocuGallo JE, Lennerstrand G. A population based study of ocu-
lar abnormalities in premature children aged 5 to 10 years. lar abnormalities in premature child

7 Keith CG, Kitchen WH. Ocular morbidity in infants of very low birthweight. Br F Ophthalmol 1983;67:302-5.

8 Quinn.GE, Dobson V, et al, on behalf of the Cryotherapy for Retinopathy of Prematurity Cooperative Group. Development of myopia in infants with birthweights less than 1251 grams. Ophthalmology 1992;99:329-40.

9 Fledelius HC. Ophthalmic changes from age of 10 to 18 years: A longitudinal study of sequels to low birth weight. II. Visual acuity. Acta Ophthalmologica 1981;59:64-70.

10 Sebris SL, Dobson V, Hartmann EE. Assessment and prediction of visual acuity in 3 to 4 year old children born prior to term. Hum Neurobiol 1984;3:87-92.

11 Page JM, Schneeweiss S, Whyte HEA, Harvey P. Ocular sequelae in premature infants. Pediatrics 1993:92:787-90. sequelae in premature infants. Pediatrics 1993;92:787-90. issenkorn I, Yassur D, Mashkowski D, Sherf I, Ben-Sira I. Myopia in premature babies with and without retinopathy
of prematurity. Br $\mathcal{F}$ Ophthalmol 1983;67:170-3. 3 Kushner BJ. Strabismus and amblyopia associated with regressed retinopathy of prematurity. Arch Ophthalmol 1982;100:256-61. 
14 Snir M, Nissenkorn I, Sherf I, Cohen S, Ben-Sira I. Visual acuity, strabismus, and amblyopia in premature babies with and without retinc
mol 1988;20:256-8.

15 Robinson R, O'Keefe M. Follow up study on premature infants with and without retinopathy of prematurity. $\mathrm{Br} \mathcal{F}$ Ophthalmol 1993;77:91-4

16 Eken P, De Vries LS, Van der Graaf Y, Meiners LC, Van Nieuwenhuizen O. Haemorrhagic -ischaemic lesions ofthe neonatal brain: Correlation between cerebral visual impairment, neurodevelopmental outcome and MRI in infancy. Dev Med Child Neurol 1995;37:41-55.

17 Hungerford J, Stewart A, Hope P. Ocular sequelae of preterm birth and their relation to ultrasound evidence of cerebral damage. Br f Ophthalmol 1986;70:463-8.

18 Abramov I, Hailine L, Lemerise E, Brown AK. Changes in visual functions of children exposed as infants to
prolonged illumination. $\mathcal{F} A m$ Optometric Assoc prolonged illum

19 Fielder AR, Moseley MJ, Ng YK. The immature visual system and premature birth. Br Med Bull 1988;44:1093-118.

20 Marlow N, Roberts BL, Cooke RWI. Outcome at 8 years for children with birthweights of $1250 \mathrm{~g}$ or less. Arch Dis Child 1993;68:286-90.

21 Davies AM. Motor and attention skills in 6 year old extremely low birthweight infants $(<1500 \mathrm{~g})$ [Thesis]. University of Liverpool, 1992.
22 TNO test for stereoscopic vision. 9th edn. Utrecht: Lameris Ootech. 1992.

23 Ginsburg AP. Functional acuity contrast test (FACT). Chicago IL: Stereooptical Co. Inc, 1993.

24 Henderson SE, Sugden DA. Movement Assessment Battery for Children. London: The London Psychological Corporation, 1992.

25 Wechsler D. Wechsler intelligence scale for children. Third edn (WISC III). San Antonio, TX: The Psychological Corporation, 1992.

26 Keen DV, Pearse RG. Birthweight between 14 and 42 weeks gestation. Arch Dis Child 1985;60:440-6.

27 Smith VH. A survey of strabismus in cerebral palsy. In: Visual disorders in cerebral palsy. Little Club Clinics in Developmental Medicine. No 9. London: Spastics Society/ opmental Medicine. No

28 Powls A, Botting N, Cooke RWI, Marlow N. Motor impairment in children 12 to 13 years old with a birthweight of less than 1250 g. Arch Dis Child 1995;72:F62-F6.

29 Haegerstrom-Portnoy G. New procedures for evaluating vision functions of special populations. Optometry and vision functions of special popu
Vision Science 1993;70:306-14.

30 Fielder AR, Robinson J, Shaw DE, Ng YK, Moseley MJ. Light and retinopathy of prematurity: Does retinal location offer a clue? Pediatrics 1992;89:648-53. 